\title{
MicroRNA-301a-3p increases oxidative stress, inflammation and apoptosis in ox-LDL-induced HUVECs by targeting KLF7
}

\author{
HUIQIONG JIANG and JIAREN LV \\ Cardiac Function Examination Room, Quanzhou First Hospital, Quanzhou, Fujian 362000, P.R. China
}

Received September 22, 2020; Accepted January 8, 2021

DOI: $10.3892 /$ etm.2021.10001

\begin{abstract}
Arteriosclerotic cardiovascular disease is an inflammatory disease of ischemia or endothelial dysfunction caused by atherosclerosis, thereby causing high mortality. The viability and apoptosis of human umbilical vein endothelial cells (HUVECs) following oxidized low-density lipoprotein (ox-LDL) induction or transfection was detected by Cell Counting Kit-8 (CCK-8) assay and flow cytometry analysis. MicroRNA (miR)-301a-3p and Krueppel-like factor 7 (KLF7) mRNA expression was determined by reverse transcription-quantitative PCR (RT-qPCR). The levels of monocyte chemoattractant protein-1 (MCP-1) and IL-6, activities of reactive oxygen species and superoxide dismutase and lactate dehydrogenase leakage were analyzed by respective commercial assay kits. The protein expression of IL-6, MCP-1, Bcl2, Bax, poly (ADP-ribose) polymerase (PARP), cleaved PARP, pro-caspase 3 and cleaved caspase- 3 was detected by western blotting. miR-301a-3p expression is highly expressed in ox-LDL-induced HUVECs. miR-301a-3p is also a target of KLF7. Inhibition of miR-301a-3p suppressed oxidative stress, inflammation and apoptosis in ox-LDL-induced HUVECs, which was reversed by KLF7 inhibition. In conclusion, miR-301a-3p promotes oxidative stress, inflammation and apoptosis in ox-LDL-induced HUVECs via decreasing KLF7 expression.
\end{abstract}

\section{Introduction}

Arteriosclerotic cardiovascular disease (ASCVD) is a chronic common inflammatory disease mediated by the immune response, and is closely associated with cytokine imbalance and inflammatory cell activation (1). ASCVD-associated mortalities account for $>40 \%$ of all household disease mortalities, and

Correspondence to: Dr Jiaren Lv, Cardiac Function Examination Room, Quanzhou First Hospital, 250 Dong Jie Road, Licheng, Quanzhou, Fujian 362000, P.R. China

E-mail: lvjiareng202@163.com

Key words: microRNA-301a-3p, oxidative stress, inflammation, apoptosis, oxidized low-density lipoprotein-induced human umbilical vein endothelial cells are one of the most important factors in the global morbidity and mortality of different diseases (2). ASCVD is caused by atherosclerosis, a complex disease associated with several risk factors, such as hypertension, diabetes inflammation and low-density lipoprotein cholesterol (3). Currently, the molecular mechanism underlying atherosclerosis remains unclear, and is considered to be associated with multiple factors, such as migration of smooth muscle cells to the intima, blood lamina activation, endothelial dysfunction and abnormal production of inflammatory factors (4), among which vascular endothelial cells have important value in maintaining the normal structure and function of blood vessels (5). Thus, understanding how to protect vascular endothelial cells is important to improve atherosclerosis, thereby decreasing the risk of ASCVD.

MicroRNAs (miRNAs/miRs) are non-coding RNA molecules that are involved in the occurrence and development of atherosclerosis plaques by regulating endothelial cell inflammation to activate the phenotypic transformation of macrophages (6). In RAW264.7 cells, miR-301a-3p affected p65 activity by regulating the expression of $N F-\kappa B$ repressing factor (NKRF), thus regulating the mRNA expression levels of TNF- $\alpha$, IL- 6 and monocyte chemoattractant protein-1 (MCP-1) (7). High levels of inflammatory factors impair vascular endothelial function and promote vascular endothelial cells to secrete adhesion factors, resulting in the development of atherosclerosis (7). Notoginsenoside R1 suppresses the NF- $\kappa$ B pathway to alleviate inflammatory injury of ATDC5 cells induced by lipopolysaccharide (LPS) by downregulating miR-301a expression (8). Overexpression of miR-301a-3p, induced by the Japanese encephalitis virus, promotes the inflammatory response by inhibiting NKRF production (9). Inhibition of miR-301a-3p relieved LPS-induced chondrogenic cell injury by activating the $\mathrm{NF}-\kappa \mathrm{B}$ signaling pathway (10). Thus, it was speculated that miR-301a-3p may also affect inflammation in atherosclerosis.

In the present study, the StarBase database (http://starbase. sysu.edu.cn/index.php) identified the ubiquitous Krueppel-like factor 7 (KLF7) as a target of miR-301a-3p. KLF7 is highly expressed in several human tissues (11). The present study aimed to determine whether miR-301a-3p promoted oxidative stress, inflammation and apoptosis in oxidized low-density lipoprotein (ox-LDL)-induced human umbilical vein endothelial cells (HUVECs) by decreasing KLF7 expression, aiming to provide the theoretical basis for the clinical treatment of ASCVD. 


\section{Materials and methods}

Cell culture and cell induction. HUVECs were purchased from the American Type Culture Collection and maintained in DMEM supplemented with $10 \%$ fetal bovine serum, $100 \mathrm{~g} / \mathrm{ml}$ streptomycin and $100 \mathrm{U} / \mathrm{ml}$ penicillin (all from Thermo Fisher Scientific, Inc.) at $37^{\circ} \mathrm{C}$ in $5 \% \mathrm{CO}_{2}$ and relative saturated humidity. Cells were cultured until fusion and subsequently passaged at a 1:3 ratio. Cells were used for experiments once they reached the logarithmic growth phase. HUVECs were treated with ox-LDL (Bioss) at different concentrations (10, $20,50,100$ and $200 \mathrm{mg} / \mathrm{l})$ for $24 \mathrm{~h}$.

Cell transfection. Following treatment of ox-LDL for $24 \mathrm{~h}$, ox-LDL-induced HUVECs were inoculated into six-well plates until $80 \%$ confluence. HUVECs were respectively transfected with miRNA inhibitor-negative control (NC; 5 nM; cat. no. miR2N0000001-1-5; Guangzhou RiboBio Co., Ltd.), miR-301a-3p inhibitor (5 nM; cat. no. miR20000688-1-5; Guangzhou RiboBio Co., Ltd.), miRNA mimic-NC (5 nM; cat. no. miR1N0000001-1-5; Guangzhou RiboBio Co., Ltd.), miR-301a-3p mimic (5 nM; cat. no. miR10000688-1-5; Guangzhou RiboBio Co., Ltd.), small interfering RNA (si) R-NC (5 nM; cat. no. siN0000002-1-5; Guangzhou RiboBio Co., Ltd.) and siKLF7 (5 nM; cat. no. siG000008609A-1-5; Guangzhou RiboBio Co., Ltd.) using Lipofectamine ${ }^{\circledR} 2000$ transfection reagent (Invitrogen; Thermo Fisher Scientific, Inc.) for $48 \mathrm{~h}$ at $37^{\circ} \mathrm{C}$. Subsequent experiments were conducted $48 \mathrm{~h}$ after transfection.

Reverse transcription-quantitative ( $R T-q) P C R$. Following treatment of ox-LDL for $24 \mathrm{~h}$, total RNA was extracted from HUVECs using TRIzol ${ }^{\circledR}$ reagent (Invitrogen; Thermo Fisher Scientific, Inc.). Total RNA (1 $\mu \mathrm{g})$ was reverse transcribed into cDNA with the Transcriptor First Strand cDNA Synthesis kit (Roche Molecular Diagnostics) at $42^{\circ} \mathrm{C}$ for $30 \mathrm{~min}$ (Takara Biotechnology Co., Ltd.). qPCR was subsequently performed using SYBR Green Master Mix (Beyotime). The following thermocycling conditions were used for the qPCR: Initial denaturation at $95^{\circ} \mathrm{C}$ for $30 \mathrm{sec}$; followed by 35 cycles of $95^{\circ} \mathrm{C}$ for $20 \mathrm{sec}, 60^{\circ} \mathrm{C}$ for $30 \mathrm{sec}$ and $72^{\circ} \mathrm{C}$ for $40 \mathrm{sec}$. The following primer pairs were used for the qPCR: miR-301a-3p forward, 5'-ACACTCCAGCTGGGCAGTGCAATAGTATTGTC-3' and reverse, 5'-CTCAACTGGTGTCGTGGA-3'; U6 forward, 5'-CTCGCTTCGGCAGCACA-3' and reverse 5'-AACGCT TCACGAATTTGCGT-3'; KLF7 forward, 5'-TTCCTGGCA GTCATCTGCAC-3' and reverse, 5'-GGGTCTGTTTGTTTG TCAGTCTGTC-3' and GAPDH forward, 5'-CCACAGTCC ATGCCATCAC-3' and reverse, 5'-GCTTCACCACCT TCTTGATG-3'. Relative expression levels were calculated using the $2^{-\Delta \Delta C q}$ method (12) and normalized to the internal reference gene GAPDH and U6.

Cell Counting Kit-8 (CCK-8) assay. Following treatment of ox-LDL for $24 \mathrm{~h}$, HUVECs were seeded into 96-well plates $\left(5 \times 10^{4}\right.$ cells/well) and incubated with $10 \mu 1$ CCK- 8 solution (Beyotime Institute of Biotechnology) at $37^{\circ} \mathrm{C}$ for $4 \mathrm{~h}$. The blank control well was zeroed, and the absorbance was measured at a wavelength of $450 \mathrm{~nm}$.
Lactate dehydrogenase ( $L D H)$ leakage. Following ox-LDL induction or transfection for $24 \mathrm{~h}$, HUVECs were seeded into 96-well plates $\left(5 \times 10^{4}\right.$ cells/well) and LDH leakage was assessed using the LDH assay kit (cat. no. C0016; Beyotime Institute of Biotechnology). The supernatant was collected, and the absorbance was measured at a wavelength of $450 \mathrm{~nm}$ using a Thermomax microplate reader (Thermo Fisher Scientific, Inc.). The LDH leakage rate was calculated as follows: $\mathrm{LDH}$ leakage $(\%)=\left(\mathrm{OD}_{\text {treatment }}-\mathrm{OD}_{\text {control }}\right) /\left(\mathrm{OD}_{\text {standard }}-\mathrm{OD}_{\text {blank }}\right)$.

Detection of MCP-1 and IL-6. Following ox-LDL induction or transfection for $24 \mathrm{~h}$, the supernatant was collected, and MCP-1 and IL-6 secretion were measured using MCP-1 (cat. no DCP00) and IL-6 (cat. no. D6050) ELISA kits (both purchased from R\&D Systems, Inc.).

Reactive oxygen species (ROS) activity. Intracellular generation of ROS was assessed via the 2',7'-dichlorofluorescin diacetate (DCFH-DA) assay (Beyotime Institute of Biotechnology). HUVECs were incubated with DCFH-DA at $37^{\circ} \mathrm{C}$ for $30 \mathrm{~min}$ in the dark. Fluorescence was subsequently measured using a fluorescence microplate reader.

Superoxide dismutase (SOD) activity. HUVECs maintained in PBS were centrifuged at $1,500 \mathrm{xg}$ for $10 \mathrm{~min}$ at $4^{\circ} \mathrm{C}$. SOD activity in the supernatant was subsequently detected using the SOD assay kit (cat. no. S0086; Beyotime Institute of Biotechnology) at a wavelength of $450 \mathrm{~nm}$ using a Thermomax microplate reader (Thermo Fisher Scientific, Inc.).

Flow cytometric analysis. Following ox-LDL induction or transfection for $24 \mathrm{~h}$, HUVECs were digested using trypsin (Beyotime Institute of Biotechnology) and subsequently adjusted to $1 \times 10^{6}$ cells $/ \mathrm{ml}$ using ice pre-cooled PBS. The cell suspension $(1 \mathrm{ml})$ was centrifuged at $1,500 \mathrm{x} \mathrm{g}$ for $10 \mathrm{~min}$ at $4^{\circ} \mathrm{C}$ and the cell precipitate was collected, which was added to $200 \mu 1$ binding buffer (Beyotime Institute of Biotechnology), $5 \mu$ Annexin V-FITC (Beyotime Institute of Biotechnology) and $5 \mu \mathrm{l}$ propidium iodide (Beyotime Institute of Biotechnology) at room temperature for $15 \mathrm{~min}$. Binding buffer $(300 \mu \mathrm{l})$ was added to each flow tube. A flow cytometry software (BD FACSDiva software v6.1.3; BD Biosciences) and CYTOMICS FC 500 flow cytometer (Beckman Coulter, Inc.) were used to detect cell apoptosis within $1 \mathrm{~h}$.

Western blotting. Following ox-LDL induction or transfection for $24 \mathrm{~h}$, HUVECs were treated with RIPA lysate (Beyotime Institute of Biotechnology), which were centrifuged at $1,500 \mathrm{x}$ g for $10 \mathrm{~min}$ at $4^{\circ} \mathrm{C}$ to extract total protein. Total protein was quantified using a BCA kit (cat. no. P0012S; Beyotime Institute of Biotechnology). A total of $30 \mu \mathrm{g}$ protein/lane was separated by $10 \%$ SDS-PAGE. The separated proteins were transferred onto polyvinylidene fluoride membranes and blocked with $5 \%$ skim milk powder for $1 \mathrm{~h}$ at room temperature. The membranes were incubated with primary antibodies against: IL-6 (cat. no. ab233706; 1:1,000; Abcam), MCP-1 (cat. no. ab214819; 1:1,000; Abcam), Bcl2 (cat. no. ab182858; 1:2,000; Abcam), Bax (cat. no. ab32503; 1:1,000; Abcam), poly (ADP-ribose) polymerase (PARP; cat. no. ab191217; 1:1,000; Abcam), cleaved PARP (cat. no. ab32064; 1:1,000; Abcam), 

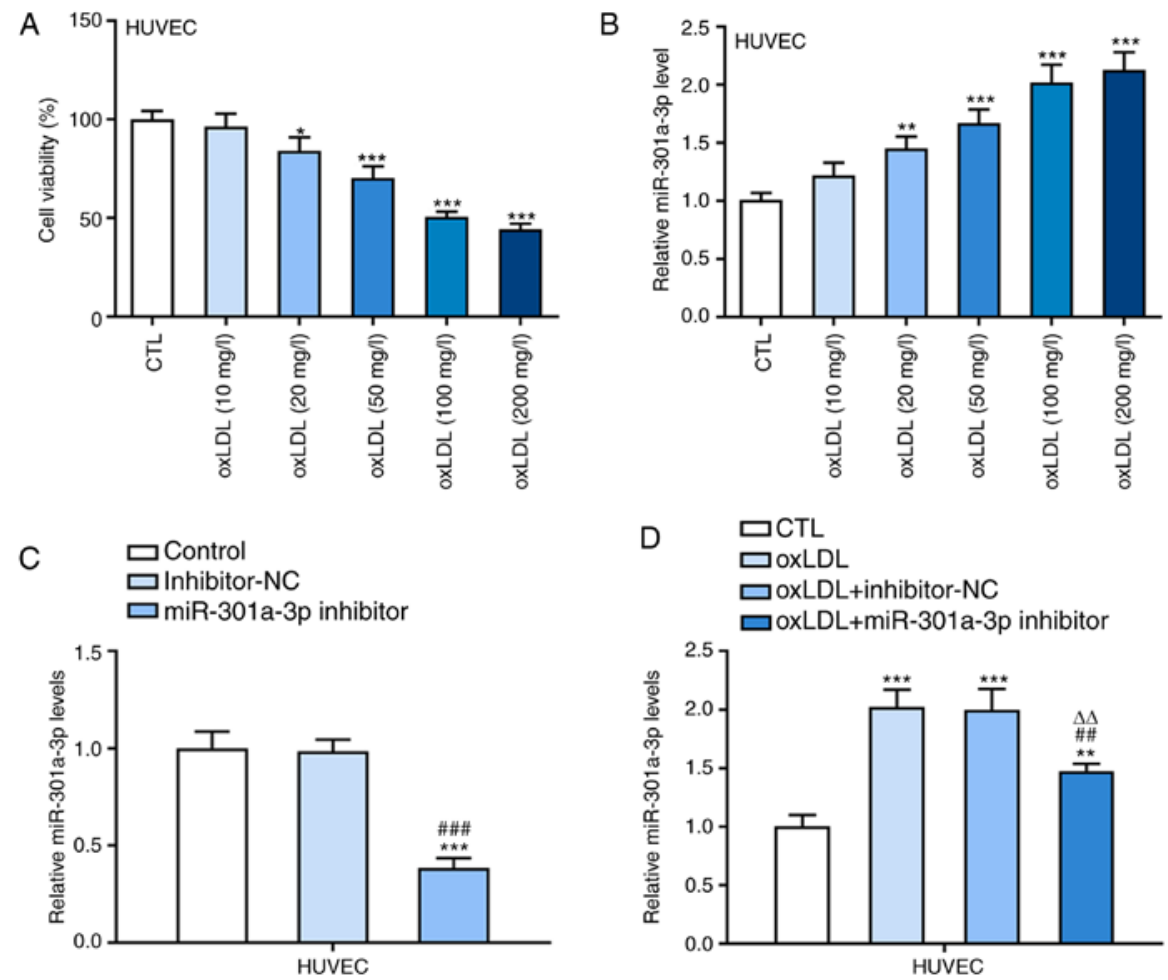

Figure 1. miR-301a-3p is highly expressed in ox-LDL-induced HUVECs. (A) The viability of HUVECs induced by different concentrations of ox-LDL was detected by Cell Counting Kit- 8 assay. ${ }^{*} \mathrm{P}<0.05$ and ${ }^{* * * *} \mathrm{P}<0.001$ vs. CTL group. (B) miR-301a-3p expression in HUVECs induced by different concentrations of ox-LDL was analyzed by RT-qPCR. ${ }^{* *} \mathrm{P}<0.01$ and ${ }^{* * *} \mathrm{P}<0.001$ vs. CTL group. (C) miR-301a-3p expression in HUVECs transfected with miR-301a-3p inhibitor was analyzed by RT-qPCR. ${ }^{* * * *} \mathrm{P}<0.001 \mathrm{vs}$. control group; ${ }^{\# \#} \mathrm{P}<0.001$ vs. inhibitor-NC group. (D) miR-301a-3p expression in HUVECs treated with ox-LDL and transfected with miR-301a-3p inhibitor was analyzed by RT-qPCR. ${ }^{* *} \mathrm{P}<0.01$ and ${ }^{* * * *} \mathrm{P}<0.001$ vs. CTL group. ${ }^{\# \# P<0.01 ~ v s . ~ o x L D L ~ g r o u p . ~}{ }^{\Delta \Delta} \mathrm{P}<0.01$ vs. oxLDL + miR-NC group. miR, microRNA; HUVEC, human umbilical vein endothelial cells; oxLDL, oxidized low-density lipoprotein; CTL, control; NC, negative control; RT-qPCR, reverse transcription-quantitative PCR.

pro-caspase3 (cat. no. ab32150; 1:1,000; Abcam), cleaved caspase-3 (cat. no. ab32042; 1:500; Abcam) and GAPDH (cat. no. ab8245; 1:1,000; Abcam) overnight at $4^{\circ} \mathrm{C}$. Membranes were washed with PBS three times and subsequently incubated with horseradish peroxidase-conjugated secondary antibody (cat. no. 7074; 1:1,000; Cell Signaling Technology, Inc.) at room temperature for $1 \mathrm{~h}$. Protein bands were visualized using ECL reagent (EMD Millipore) and imaged on a Chemiluminescence Imaging system (Tanon Science and Technology Co., Ltd.). Image-Pro Plus software (version 6.0; Media Cybernetics, Inc.) was used for densitometry.

Dual-luciferase reporter assay. HUVECs were co-transfected with pGL3-KLF7 3'-untranslated region (UTR) plasmid (containing mutant KLF7 3'-UTR or wild-type KLF7 3'-UTR) and miR-301a-3p mimic or miR-NC vector (Biovector NTCC Inc.) using Lipofectamine ${ }^{\circledR} 2000$ reagent. Following incubation for $48 \mathrm{~h}$ at $37^{\circ} \mathrm{C}$, a Dual-Luciferase ${ }^{\circledR}$ Reporter Assay system (Promega Corporation) was used to detect relative luciferase activities, which was normalized to Renilla luciferase activity.

Statistical analysis. Three separate experiments were repeated for each experiment. Statistical analysis was performed using SPSS 22.0 software (IBM Corp.). Data are presented as the mean \pm SD. Unpaired Student's t-test was used to compare differences between two groups, while one-way ANOVA and Tukey's post hoc test were used to compare differences between multiple groups. $\mathrm{P}<0.05$ was considered to indicate a statistically significant difference.

\section{Results}

miR-301a-3p is highly expressed in ox-LDL-induced HUVECs. Following treatment with ox-LDL at different concentrations, the viability of HUVECs gradually decreased upon decreasing ox-LDL concentration (10-200 mg/l; Fig. 1A). Furthermore, miR-301a-3p expression gradually increased in ox-LDL-induced HUVECs treated with 10-100 mg/l ox-LDL (Fig. 1B). No significant differences were observed in cell viability and miR-301a-3p expression between $100 \mathrm{mg} / \mathrm{l}$ ox-LDL-induced HUVECs and $200 \mathrm{mg} / \mathrm{l}$ ox-LDL-induced HUVECs. Hence, $100 \mathrm{mg} / \mathrm{l}$ ox-LDL was selected for subsequent experimentation. miR-301a-3p expression level was notably decreased in HUVECs transfected with miR-301a-3p inhibitor (Fig. 1C). As presented in Fig. 1D, miR-301a-3p expression significantly increased in ox-LDL-induced HUVECs compared with controls, while transfection with miR-301a-3p inhibitor significantly downregulated miR-301a-3p expression in ox-LDL-induced HUVECs.

miR-301a-3p knockdown inhibits inflammation and oxidative stress in ox-LDL-induced HUVECs. ox-LDL significantly promoted the protein expression levels of IL-6 and MCP-1, the effects of which were reversed following miR-301a-3p knockdown (Fig. 2A). Similarly, IL-6 and MCP-1 expression 

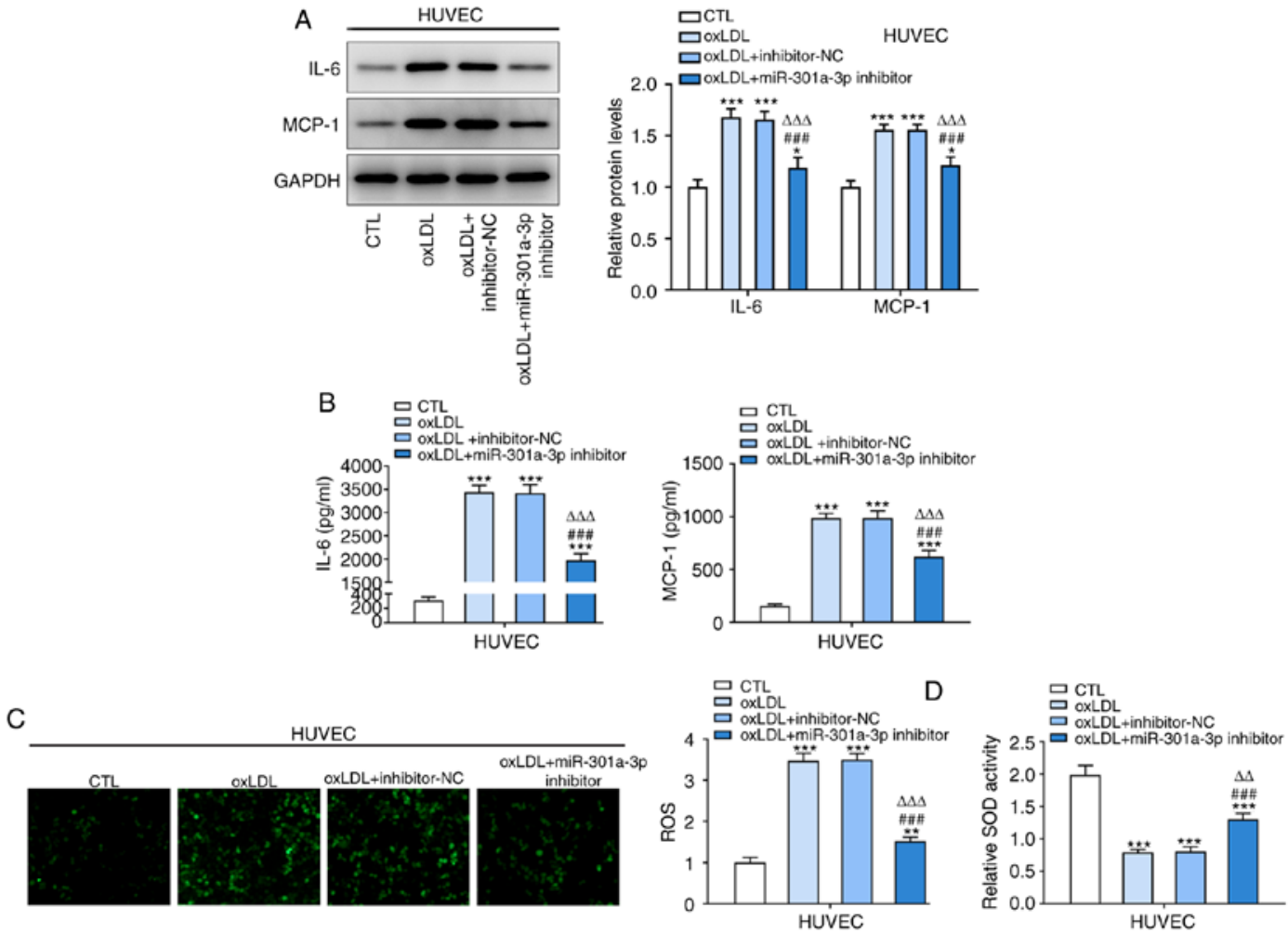

Figure 2. Inhibition of miR-301a-3p inhibits inflammation and oxidative stress in ox-LDL-induced HUVECs. (A) The expression of IL-6 and MCP-1 in HUVECs treated with ox-LDL and transfected with miR-301a-3p inhibitor was analyzed by western blot analysis. (B) The levels of IL-6 and MCP-1 in HUVECs treated with ox-LDL and transfected with miR-301a-3p inhibitor was analyzed by ELISA. (C) ROS level in HUVECs treated with ox-LDL and transfected with miR-301a-3p inhibitor was determined by a 2',7'-dichlorofluorescin diacetate assay (magnification, x200). (D) The SOD levels in HUVECs treated with ox-LDL and transfected with miR-301a-3p inhibitor was determined by a SOD assay kit. ${ }^{*} \mathrm{P}<0.05,{ }^{* *} \mathrm{P}<0.01$ and ${ }^{* * * *} \mathrm{P}<0.001$ vs. CTL group. ${ }^{\# \# \# *} \mathrm{P}<0.001$ vs. oxLDL group. ${ }^{\Delta \Delta} \mathrm{P}<0.01$ and ${ }^{\Delta \Delta \Delta} \mathrm{P}<0.01$ vs. oxLDL + miR-NC group. miR, microRNA; HUVEC, human umbilical vein endothelial cells; oxLDL, oxidized low-density lipoprotein; CTL, control; NC, negative control; MCP-1, monocyte chemoattractant protein-1; ROS, reactive oxygen species; SOD, superoxide dismutase.
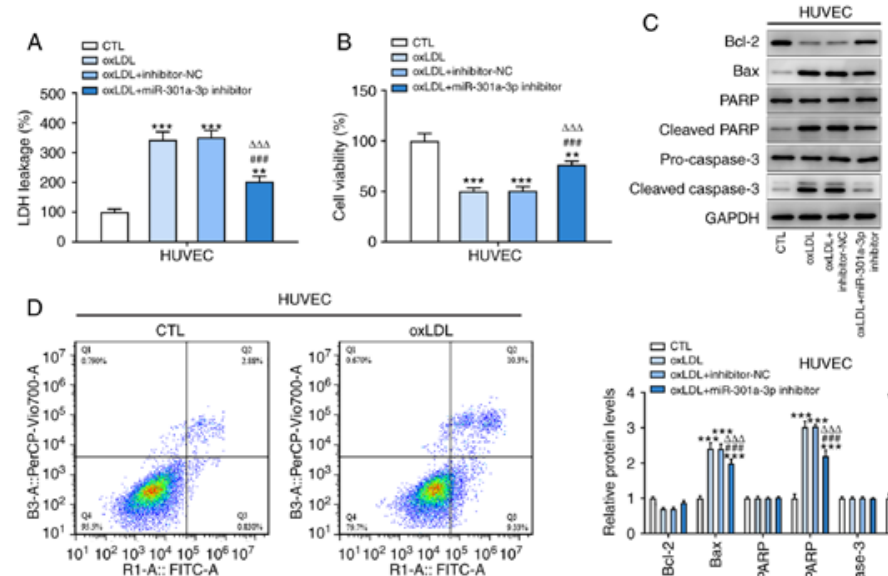

HUVEC
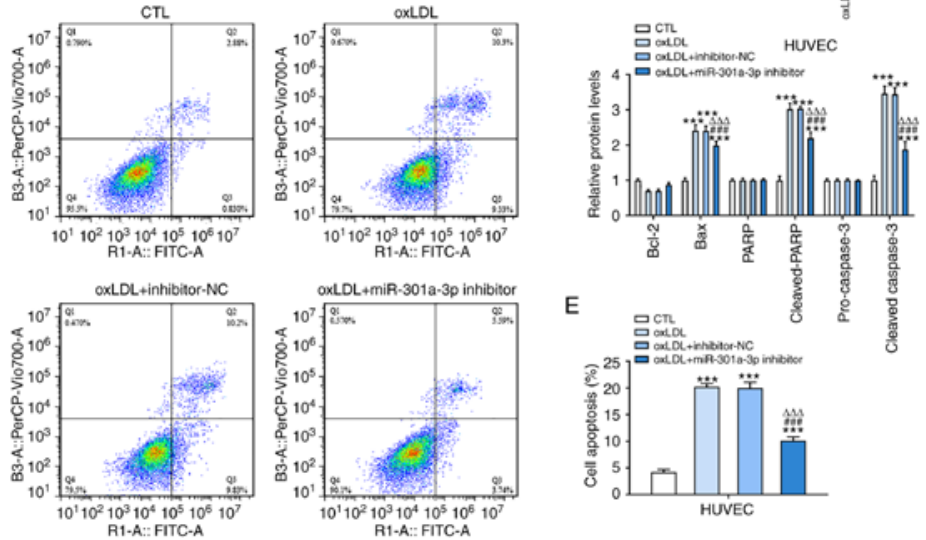

Figure 3. Inhibition of miR-301a-3p inhibits apoptosis in ox-LDL-induced HUVECs. (A) LDH leakage in HUVECs treated with ox-LDL and transfected with miR-301a-3p inhibitor was detected by an LDH assay kit. (B) The viability of HUVECs treated with ox-LDL and transfected with miR-301a-3p inhibitor was detected by Cell Counting Kit-8 assay. (C) The expression of apoptosis-related proteins in HUVECs treated with ox-LDL and transfected with miR-301a-3p inhibitor was determined by western blot analysis. (D and E) The apoptosis of HUVECs treated with ox-LDL and transfected with miR-301a-3p inhibitor was detected by flow cytometry analysis. ${ }^{* *} \mathrm{P}<0.01$ and ${ }^{* * * *} \mathrm{P}<0.001$ vs. CTL group. ${ }^{\# \# \#} \mathrm{P}<0.001$ vs. oxLDL group. ${ }^{\Delta \Delta \Delta} \mathrm{P}<0.001$ vs. oxLDL + miR-NC group. miR, microRNA; HUVEC, human umbilical vein endothelial cells; oxLDL, oxidized low-density lipoprotein; CTL, control; NC, negative control; LDH, lactate dehydrogenase; PARP, poly (ADP-ribose) polymerase. 

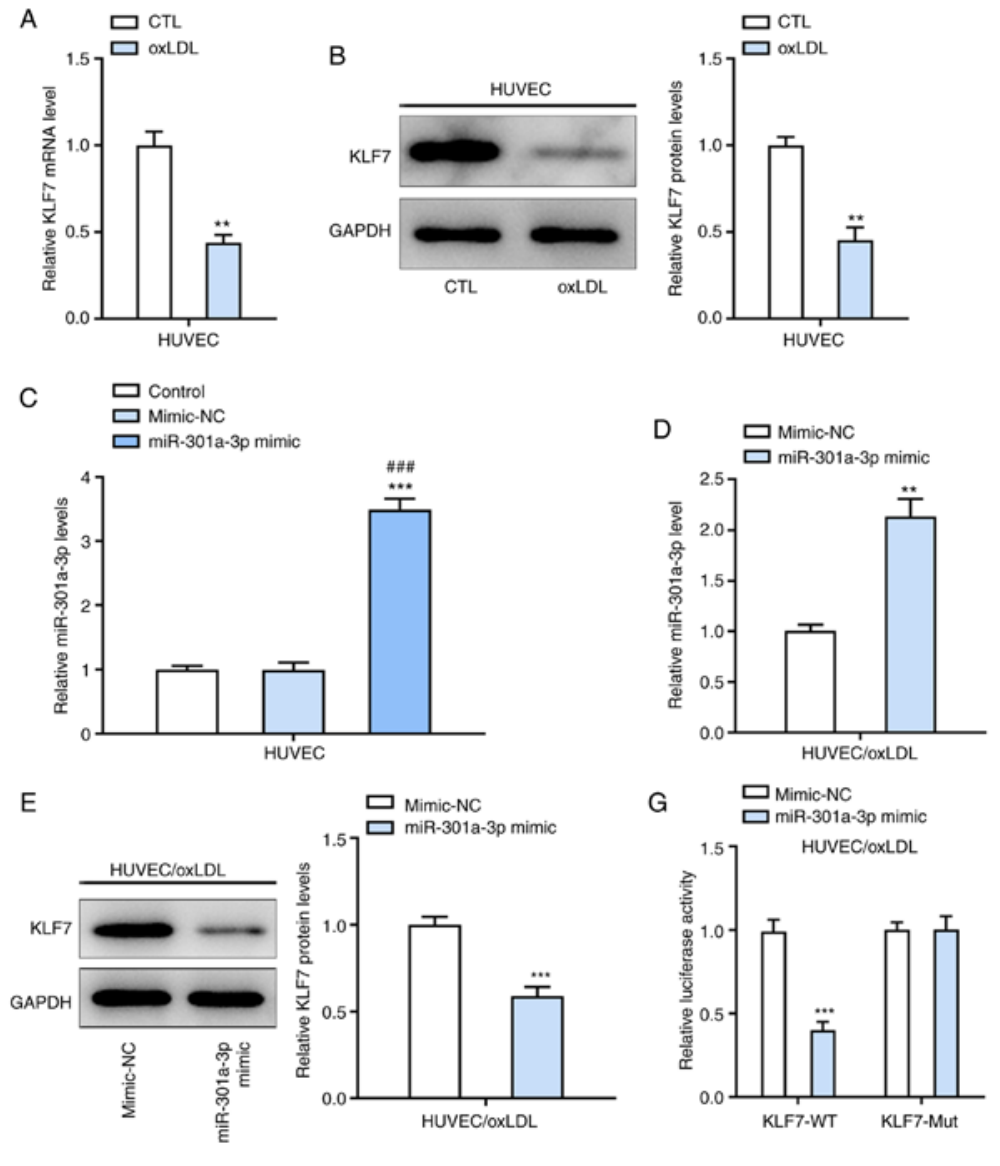

G
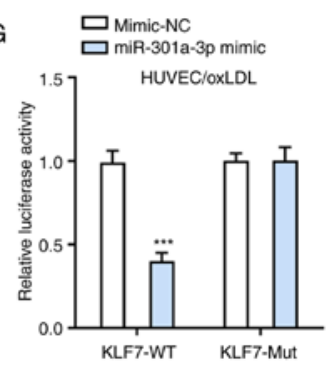

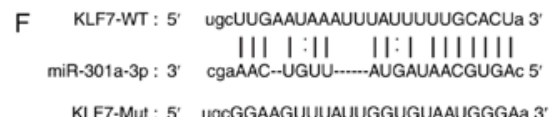

Figure 4. miR-301a-3p targets KLF7 in ox-LDL-induced HUVECs. (A) KLF7 mRNA expression in ox-LDL-induced HUVECs was analyzed by RT-qPCR. * $<<0.01$ vs. CTL group. (B) KLF7 protein expression in ox-LDL-induced HUVECs was analyzed by western blot analysis. ${ }^{* *} \mathrm{P}<0.01$ vs. CTL group. (C) miR-301a-3p expression in ox-LDL-induced HUVECs following miR-301a-3p mimic transfection was analyzed by RT-qPCR. ${ }^{* * *} \mathrm{P}<0.001$ vs. CTL group. ${ }^{\# \#} \mathrm{P}<0.001$ vs. Mimic-NC group. (D/E) KLF7 expression in ox-LDL-induced HUVECs following miR-301a-3p mimic transfection was analyzed by western blot analysis. ${ }^{* *} \mathrm{P}<0.001 \mathrm{vs.} \mathrm{Mimic-NC}$ group. (F) Binding sites between KLF7 and miR-301a-3p. (G) Luciferase reporter assay was performed to confirm the direct binding relationship between KLF7 and miR-301a-3p. ${ }^{* * *} \mathrm{P}<0.001$ vs. Mimic-NC group. miR, microRNA; HUVEC, human umbilical vein endothelial cells; oxLDL, oxidized low-density lipoprotein; CTL, control; NC, negative control; KLF7, Krueppel-like factor 7; WT, wild-type; Mut, mutant; RT-qPCR, reverse transcription-quantitative PCR.

levels increased in ox-LDL-induced HUVECs, the effects of which were reversed following miR-301a-3p knockdown (Fig. 2B). In addition, ROS and SOD levels were upregulated in ox-LDL-induced HUVECs, the effects of which were reversed following miR-301a-3p knockdown (Fig. 2C and D).

miR-301a-3p knockdown inhibits apoptosis in ox-LDL-induced HUVECs. LDH leakage was increased and cell viability was decreased in ox-LDL-induced HUVECs compared with the control group (Fig. 3A and B). In addition, the expression levels of Bax, cleaved PARP and cleaved caspase- 3 was increased while Bcl-2 expression was decreased in ox-LDL-induced HUVECs (Fig. 3C). The apoptosis ratio of ox-LDL-induced HUVECs was increased compared with controls (Fig.3D and E). In addition, miR-301a-3p knockdown reversed the aforementioned changes in HUVECs induced by ox-LDL.

miR-301a-3p targets KLF7 in ox-LDL-induced HUVECs. Both mRNA and protein levels of KLF7 were significantly decreased in ox-LDL-induced HUVECs compared with controls (Fig. 4A and B). Following transfection with miR-301a-3p mimic, miR-301a-3p expression significantly increased in ox-LDL-induced HUVECs compared with the miR-NC group (Fig. 4C). In addition, overexpression of miR-301a-3p suppressed KLF7 expression in ox-LDL-induced HUVECs (Fig. 4D and E). The binding sites between miR-301a-3p and KLF7 are presented in Fig. 4F. Relative luciferase activity significantly decreased in ox-LDL-induced HUVECs co-transfected with miR-301a-3p mimic and KLF7-WT (Fig. 4G).

Inhibition of KLF7 weakens the inhibitory effects of miR-301a-3p inhibition on inflammation and oxidative stress in ox-LDL-induced HUVECs. KLF7 expression decreased following transfection of ox-LDL-induced HUVECs with siKLF7 (Fig. 5A and B). miR-301a-3p knockdown downregulated the protein expression levels of IL- 6 and MCP-1 in ox-LDL-induced HUVECs (Fig. 5C and D). In addition, miR-301a-3p knockdown downregulated ROS expression and 

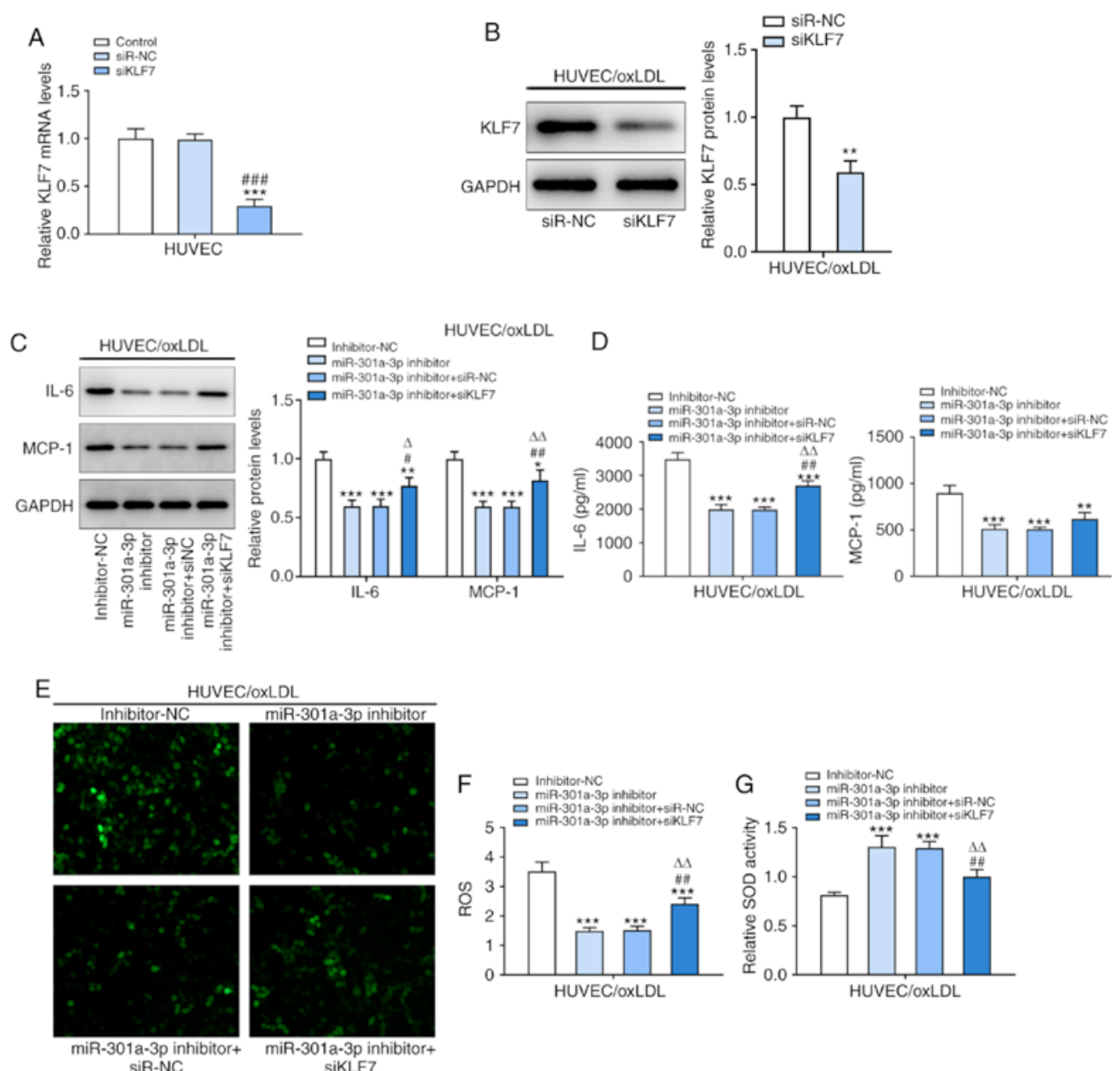

Figure 5. Inhibition of KLF7 weakens the inhibitory effects of miR-301a-3p inhibition on inflammation and oxidative stress in ox-LDL-induced HUVECs. (A) KLF7 protein expression in ox-LDL-induced HUVEC following transfection of miR-301a-3p inhibitor transfection and siKLF7 was analyzed by western blot analysis. ${ }^{* * *} \mathrm{P}<0.001$ vs. Control group. ${ }^{\# \#} \mathrm{P}<0.001$ vs. siR-NC group. (B) The expression of KLF7 in ox-LDL-induced HUVECs following transfection of siKLF7 was analyzed by western blot analysis. ${ }^{* *} \mathrm{P}<0.01$ vs. siR-NC group. (C) The levels of IL-6 and MCP-1 in ox-LDL-induced HUVECs following transfection of miR-301a-3p inhibitor and siKLF7 was analyzed by western blot analysis. (D) The levels of IL-6 and MCP-1 in ox-LDL-induced HUVECs following transfection of miR-301a-3p inhibitor and siKLF7 was analyzed by ELISA. (E and F) The ROS level in ox-LDL-induced HUVECs following transfection of miR-301a-3p inhibitor and siKLF7 was determined by a 2',7'-dichlorofluorescin diacetate assay. (G) The SOD levels in ox-LDL-induced HUVECs following transfection of miR-301a-3p inhibitor and siKLF7 was determined by a SOD assay kit. ${ }^{*} \mathrm{P}<0.05,{ }^{* * *} \mathrm{P}<0.01$ and ${ }^{* * *} \mathrm{P}<0.001$ vs. miR-NC group. ${ }^{*} \mathrm{P}<0.05$ and ${ }^{\# \#} \mathrm{P}<0.01$ vs. miR-301a-3p inhibitor group. ${ }^{\Delta} \mathrm{P}<0.05$ and ${ }^{\Delta \Delta} \mathrm{P}<0.01$ vs. miR-301a-3p inhibitor + siR-NC group. miR, microRNA; HUVEC, human umbilical vein endothelial cells; oxLDL, oxidized low-density lipoprotein; CTL, control; NC, negative control; KLF7, Krueppel-like factor 7; MCP-1, monocyte chemoattractant protein-1; ROS, reactive oxygen species; SOD, superoxide dismutase; si, small interfering.

upregulated SOD expression in ox-LDL-induced HUVECs (Fig. 5E-G). Collectively, these results suggested that inhibition of KLF7 may reverse the inhibitory effects of miR-301a-3p on ox-LDL-induced HUVECs.

Inhibition of KLF7 weakens the inhibitory effects of miR-301a-3p inhibition on apoptosis in ox-LDL-induced HUVECs. miR-301a-3p knockdown decreased LDH leakage and increased cell viability in ox-LDL-induced HUVECs (Fig. 6A and B). In addition, the expression levels of Bax, cleaved PARP and cleaved caspase-3 was decreased, while Bcl-2 expression was increased in ox-LDL-induced HUVECs transfected with miR-301a-3p inhibitor (Fig. 6C), and inhibition of miR-301a-3p suppressed the ratio of apoptosis of ox-LDL-induced HUVECs (Fig. 6D and E). In addition, inhibition of KLF7 could reverse the aforementioned changes in ox-LDL-induced HUVECs caused by miR-301a-3p inhibition.

\section{Discussion}

In the present study, an ox-LDL-induced HUVEC model was used to simulate atherosclerosis that leads to ASCVD. It was found that inhibition of miR-301a-3p suppressed oxidative stress, inflammation and apoptosis in ox-LDL-induced HUVEC, which was reversed by KLF7 inhibition. Studies have demonstrated that $>50 \%$ of cardiovascular diseases, including atherosclerosis, are inextricably associated with genetics $(13,14)$. miRNAs are involved in regulating several physiological and pathological mechanisms, including inflammation and cardiovascular disease (15).

Several studies have suggested that miRNAs play an important role in the progression of atherosclerosis and inflammatory response. For example, miR-21 expression is significantly increased in the miRNA expression profile of atherosclerosis (16). miR-21 is abundantly expressed in macrophages, the lack of which aggravates the progression 

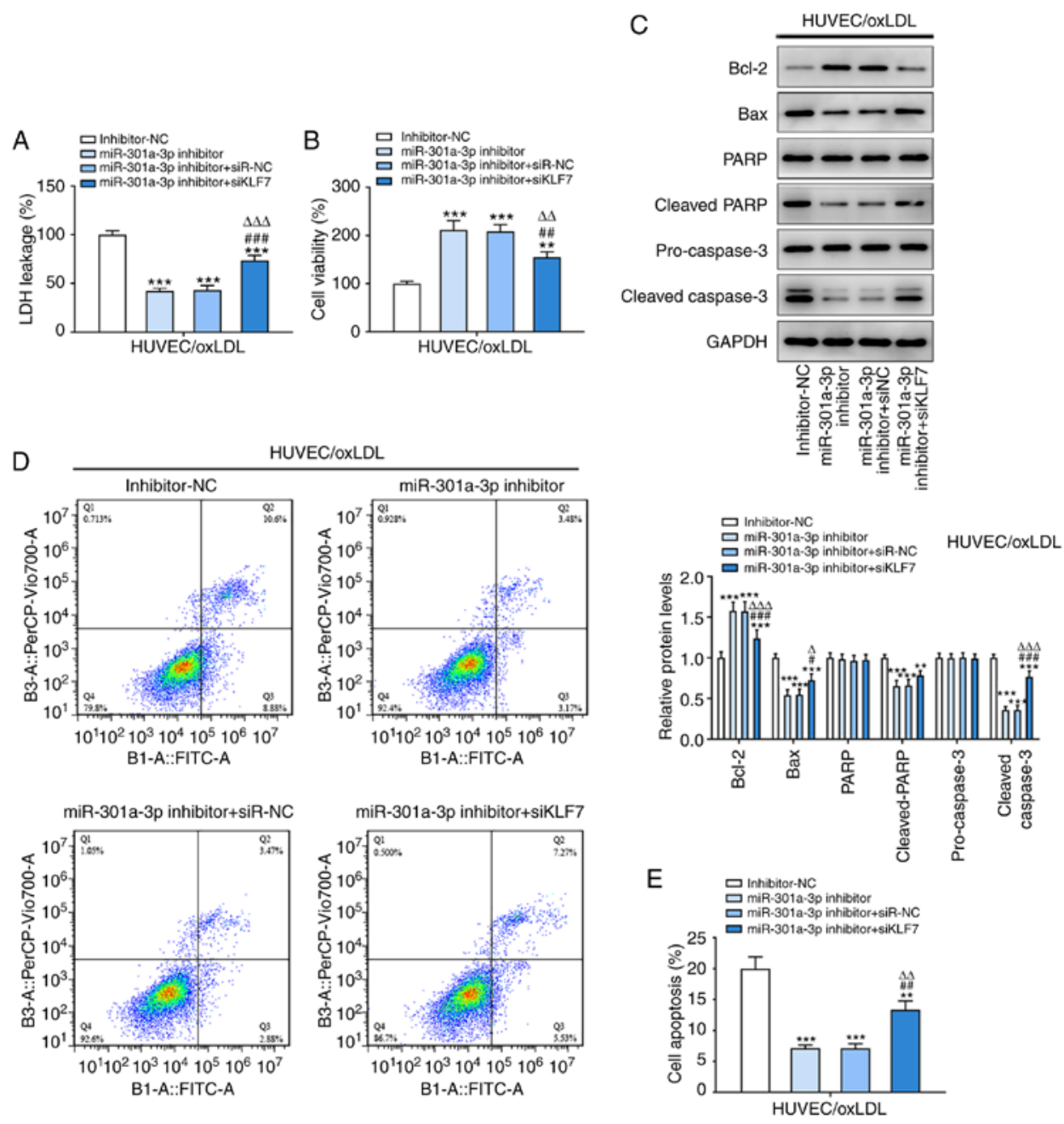

Figure 6. Inhibition of KLF7 weakens the inhibitory effects of miR-301a-3p inhibition on apoptosis in ox-LDL-induced HUVECs. (A) LDH leakage in ox-LDL-induced HUVECs following transfection of miR-301a-3p inhibitor and siKLF7 was detected by an LDH assay kit. (B) The viability of ox-LDL-induced HUVECs following transfection of miR-301a-3p inhibitor and siKLF7 was detected by Cell Counting Kit-8 assay. (C) The expression of apoptosis-related proteins in ox-LDL-induced HUVECs following transfection of miR-301a-3p inhibitor and siKLF7 was determined by western blot analysis. (D and E) The apoptosis of ox-LDL-induced HUVECs following transfection of miR-301a-3p inhibitor and siKLF7 was detected by flow cytometry analysis. ** P<0.01 and ${ }^{* * * *} \mathrm{P}<0.001$ vs. miR-NC group. ${ }^{\# \#} \mathrm{P}<0.01$ and ${ }^{\# \# \#} \mathrm{P}<0.001$ vs. miR-301a-3p inhibitor group. ${ }^{\Delta \Delta} \mathrm{P}<0.01$ and ${ }^{\Delta \Delta \Delta} \mathrm{P}<0.001$ vs. miR-301a-3p inhibitor + siR-NC group. miR, microRNA; HUVEC, human umbilical vein endothelial cells; oxLDL, oxidized low-density lipoprotein; CTL, control; NC, negative control; si, small interfering; LDH, lactate dehydrogenase; PARP, poly (ADP-ribose) polymerase.

of atherosclerosis (17). miR-21 decreased IL-6 expression via the Toll-like receptor 4 and NF- $\kappa B$ pathways, while increasing IL-10 expression to decrease LPS-induced lipid deposition in macrophages. Hence, miR-21 may reverse the bacterial infection-induced pathological process of atherosclerosis (18). In addition, severe burns inhibit miR-301a-3p expression, which suppresses the apoptosis of HUVECs and stabilizes endothelial barrier permeability (19). miR-301a-3p expression is upregulated in obesity-related inflammation, and miR-301a decreases the production of proinflammatory cytokines in 3T3-L1 cells (20). miR-301a-3p boosts intestinal mucosal inflammation by increasing the expression levels of IL-17A and TNF- $\alpha$ in inflammatory bowel disease (21). The results of the present study demonstrated that miR-301a-3p expression was increased in ox-LDL-induced HUVECs, and inhibition of miR-301a-3p suppressed oxidative stress, inflammation and apoptosis in ox-LDL-induced HUVECs.

Macrophages participate in the immune response of the vascular wall, and thus promote the formation and development of atherosclerosis (22). KLF7 expression is downregulated in some injury diseases, thus KLF7 may inhibit disease progression. Inhibition of KLF7 notably suppressed the proliferation and migration of Schwann cells (23). Adult corticospinal tract (CST) neurons failed to promote KLF7 expression due to axon injury, while overexpression of VP16-KLF7 in vivo promoted both sprouting and regenerative axon growth in the CST of adult mice (24). The results of the present study demonstrated that KLF7 expression decreased in ox-LDL-induced HUVECs, and inhibition of KLF7 reversed the effect of inhibition of miR-301a-3p and promoted oxidative stress, inflammation and apoptosis in ox-LDL-induced HUVECs.

In conclusion, the results of the present study demonstrated that miR-301a-3p expression was upregulated and KLF7 expression was downregulated in ox-LDL-induced HUVECs. In addition, miR-301a-3p induced oxidative stress, inflammation and apoptosis in ox-LDL-induced HUVECs by decreasing KLF7 expression. However, the present study only performed 
cell experiments. In vivo experiments will be conducted in the future to support the present results.

\section{Acknowledgements}

Not applicable.

\section{Funding}

This study was supported by the Fujian Provincial Health and Family Planning Project Funding Plan for Rural and Urban Communities to Promote Appropriate Technology in 2018 (grant no. 2018025).

\section{Availability of data and materials}

The datasets used and/or analyzed during the current study are available from the corresponding author on reasonable request.

\section{Authors' contributions}

JL designed the experiments. HJ performed the experiments and collected the data. $\mathrm{HJ}$ analyzed the experimental data and drafted the manuscript. JL revised the manuscript. JL and HJ confirm the authenticity of the raw data. All authors read and approved the final manuscript.

\section{Ethics approval and consent to participate}

Not applicable.

\section{Patient consent for publication}

Not applicable.

\section{Competing interests}

The authors declare they have no competing interests.

\section{References}

1. Brown WV, Remaley AT and Ridker PM: JCL Roundtable: is inflammation a future target in preventing arteriosclerotic cardiovascular disease. J Clin Lipidol 9: 119-128, 2015.

2. Zhou M, Wang H, Zeng X, Yin P, Zhu J, Chen W, Li X, Wang L, Wang L, Liu Y, et al: Mortality, morbidity, and risk factors in China and its provinces, 1990-2017: A systematic analysis for the Global Burden of Disease Study 2017. Lancet 394: 1145-1158, 2019.

3. Roth EM: Alirocumab for low-density lipoprotein cholesterol lowering. Future Cardiol 15: 17-29, 2019.

4. Khosravi M, Poursaleh A, Ghasempour G, Farhad S and Najafi M: The effects of oxidative stress on the development of atherosclerosis. Biol Chem 400: 711-732, 2019.

5. Lankin VZ, Sharapov MG, Goncharov RG, Tikhaze AK and Novoselov VI: Natural dicarbonyls inhibit peroxidase activity of peroxiredoxins. Dokl Biochem Biophys 485: 132-134, 2019.

6. de Lucia C, Komici K, Borghetti G, Femminella GD, Bencivenga L, Cannavo A, Corbi G, Ferrara N, Houser SR, Koch WJ and Rengo G: microRNA in cardiovascular aging and age-related cardiovascular diseases. Front Med (Lausanne) 4: 74, 2017.
7. Lin D, Xiu-Qing H, Tao S, Lin-Fang LI, Jian LI and Amp BH: miR-301a regulates the expression of inflammatory factors in macrophages. Chin J Arterioscler 25: 447-451, 2017.

8. Dong Y, Yan X, Yang X, Yu C, Deng Y, Song X and Zhang L: Notoginsenoside R1 suppresses miR-301a via NF- $\kappa$ B pathway in lipopolysaccharide-treated ATDC5 cells. Exp Mol Pathol 112: $104355,2020$.

9. Hazra B, Chakraborty S, Bhaskar M, Mukherjee S, Mahadevan A and Basu A: miR-301a regulates inflammatory response to japanese encephalitis virus infection via suppression of NKRF activity. J Immunol 203: 2222-2238, 2019.

10. Chen H, Qi J, Bi Q and Zhang S: Suppression of miR-301a alleviates LPS-induced inflammatory injury in ATDC5 chondrogenic cells by targeting Sirt1. Int J Clin Exp Pathol 10: 8991-9000, 2017.

11. Guan F, Kang Z, Zhang JT, Xue NN, Yin H, Wang L, Mao BB, Peng WC, Zhang BL, Liang X, et al: KLF7 promotes polyamine biosynthesis and glioma development through transcriptionally activating ASL. Biochem Biophys Res Commun 514: 51-57, 2019.

12. Livak KJ and Schmittgen TD: Analysis of relative gene expression data using real-time quantitative PCR and the 2(-Delta Delta C(T)) Method. Methods 25: 402-408, 2001.

13. Wysocka A, Cybulski M, Berbeć H, Wysokiński A, Stążka J, Daniluk $J$ and Zapolski T: Dynamic changes of paraoxonase 1 activity towards paroxon and phenyl acetate during coronary artery surgery. BMC Cardiovasc Disord 17: 92, 2017.

14. Marenberg ME, Risch N, Berkman LF, Floderus B and de Faire U: Genetic susceptibility to death from coronary heart disease in a study of twins. N Engl J Med 330: 1041-1046, 1995.

15. Schober A and Weber C: Mechanisms of MicroR NAs in Atherosclerosis. Annu Rev Pathol 11: 583-616, 2016.

16. Pordzik J, Pisarz K, De Rosa S, Jones AD, Eyileten C, Indolfi C, Malek L and Postula M: The potential role of platelet-related micrornas in the development of cardiovascular events in high-risk populations, including diabetic patients: a review. Front Endocrinol (Lausanne) 9: 74, 2018.

17. Canfrán-Duque A, Rotllan N, Zhang X, Fernández-Fuertes M, Ramírez-Hidalgo C, Araldi E, Daimiel L, Busto R, Fernández-Hernando C and Suárez Y: Macrophage deficiency of miR-21 promotes apoptosis, plaque necrosis, and vascular inflammation during atherogenesis. EMBO Mol Med 9: 1244-1262, 2017.

18. Feng J, Li A, Deng J, Yang Y, Dang L, Ye Y, Li Y and Zhang W: miR-21 attenuates lipopolysaccharide-induced lipid accumulation and inflammatory response: Potential role in cerebrovascular disease. Lipids Health Dis 13: 27, 2014.

19. Liu L, Yin H, Hao X, Song H, Chai J, Duan H, Chang Y, Yang L, Wu Y, Han S, et al: Down-Regulation of miR-301a-3p Reduces Burn-Induced Vascular Endothelial Apoptosis by potentiating hMSC-Secreted IGF-1 and PI3K/Akt/FOXO3a Pathway. iScience 23: 101383, 2020.

20. Li H, Xue M, Xu J and Qin X: MiR-301a is involved in adipocyte dysfunction during obesity-related inflammation via suppression of PPAR $\gamma$. Pharmazie 71: 84-88, 2016.

21. He C, Shi Y, Wu R, Sun M, Fang L, Wu W, Liu C, Tang M, Li Z, Wang P, et al: miR-301a promotes intestinal mucosal inflammation through induction of IL-17A and TNF- $\alpha$ in IBD. Gut 65: 1938-1950, 2016.

22. Domínguez-Andrés J and Netea MG: Long-term reprogramming of the innate immune system. J Leukoc Biol 105: 329-338, 2019.

23. Li WY, Zhang WT, Cheng YX, Liu YC, Zhai FG, Sun P, Li HT, Deng LX, Zhu XF and Wang Y: Inhibition of KLF7-Targeting MicroRNA 146b Promotes Sciatic Nerve Regeneration. Neurosci Bull 34: 419-437, 2018.

24. Blackmore MG, Wang Z, Lerch JK, Motti D, Zhang YP, Shields CB, Lee JK, Goldberg JL, Lemmon VP and Bixby JL: Krüppel-like Factor 7 engineered for transcriptional activation promotes axon regeneration in the adult corticospinal tract. Proc Natl Acad Sci USA 109: 7517-7522, 2012.

This work is licensed under a Creative Commons Attribution-NonCommercial-NoDerivatives 4.0 International (CC BY-NC-ND 4.0) License. 